\title{
THE IMPORTANCE OF SHORT ADRENERGIC NEURONS IN THE SEMINAL EMISSION MECHANISM OF RAT, GUINEA-PIG AND MAN
}

\author{
CH. OWMAN AND N.-O. SJÖBERG \\ Institute of Anatomy and Histology and Department of Obstetrics and Gynaecology \\ at Malmö, University of Lund, Sweden
}

(Received 16th March 1971, accepted 5th Fuly 1971)

\begin{abstract}
Summary. The trigonum area of the bladder-including the bladder neck and the proximal urethra-of rats and guinea-pigs contains large amounts of noradrenaline. This is consistent with a rich adrenergic innervation of the smooth musculature, particularly in the internal sphincter of the bladder. The innervation is not reduced by hypogastric denervation, indicating that it originates from peripheral ganglia (i.e. short adrenergic neurons). Stimulation of the hypogastric nerves produces seminal emission into the proximal urethra. After hypogastric denervation, there is a seminal reflux into the bladder whether copulation has occurred or not.

Extensive presacral operative dissection in male patients usually results in seminal reflux into the bladder. The excised tissues often contain adrenergic ganglion formations, probably identical with the short adrenergic neurons.

It is suggested that removal of these ganglia, or their more proximal sympathetic input, results in permanent motor deficiency of the smooth musculature in the structures engaged in the emission mechanism, including the bladder neck. This results in a continuous seminal reflux into the bladder (whether coitus occurs or not) due to a continuous slow transport of seminal fluid through the vas deferens as a consequence of persisting segmental contraction and continuous secretion from the accessory genital glands.
\end{abstract}

\section{INTRODUCTION}

In spite of the early suggestion by Langley \& Anderson (1895b, 1896) that some of the sympathetic nerve fibres to the pelvic viscera form synapses in ganglia near the effector organs, the generally accepted opinion has long been that the hypogastric nerves innervate the accessory male genital organs by postganglionic fibres which arise in the inferior mesenteric ganglia. However, the motor response of the vas deferens to hypogastric nerve stimulation has been shown to be inhibited by ganglionic blocking agents (Sjöstrand, 
1962a) and sectioning of the hypogastric nerves does not overtly reduce the noradrenaline content of the internal male genital organs (Sjöstrand, 1962b), confirming the existence of a peripheral relay. Fluorescence histochemistry has revealed that the smooth musculature of the vas deferens, seminal vesicles and prostate of several species, including man, receives an extensive adrenergic innervation, and large formations of adrenergic ganglion cells have been demonstrated within or close to these organs (Falck, Owman \& Sjöstrand, 1965; Owman \& Sjöstrand, 1965; Sjöstrand, 1965; Baumgarten, Falck, Holstein, Owman \& Owman, 1968). Such 'short' adrenergic neurons have also been found to innervate the smooth musculature in the trigonum area of the urinary bladder (Hamberger \& Norberg, 1965a, b) and the urethra (Owman, Owman \& Sjöberg, 1971).

Semans \& Langworthy (1938) have shown that the emission of the seminal fluid, i.e. its transport into the pelvic urethra, is controlled by the hypogastric nerves, whereas ejaculation, i.e. the discharge of seminal fluid from the urethra through the external urethral meatus, is mainly effected by the parasympathetic internal pudendal nerve. Since the systems of short adrenergic neurons innervate not only the male internal genital organs but also the urethra and trigonum, it can be assumed that these neurons are directly involved in the emission mechanism, and that they are affected in certain conditions of sterility resulting from seminal reflux into the urinary bladder (Goligher, 1951; Hotchkiss, Pinto \& Kleegman, 1955; Walters \& Kaufman, 1959; Schellen, 1960; Rieser, 1961; Gennser, Owman, Owman \& Wehlin, 1969). These possibilities have been the subject of the present experimental study on rats and guinea-pigs.

\section{MATERIALS AND METHODS}

Rats, guinea-pigs and tissues from human subjects were used in the following studies.

\section{Rat experiments}

Sixty-three male and sixty female Sprague-Dawley rats, of about $250 \mathrm{~g}$ body weight, were allowed free access to a standard pellet diet (SAN-bolagen, Sweden) and tap water.

(1) Five of the male rats were subjected to hypogastric denervation under ether anaesthesia. The abdomen was opened through a midline incision, about $15 \mathrm{~mm}$ of each hypogastric nerve was removed and the incision was closed. The animals were killed by decapitation 1 week later. Five intact animals served as controls. The trigonum area of the bladder together with its neck and the proximal part of the urethra was removed in one piece from the level of the ureteric ostia down to the colliculus seminalis. The preparation was frozen to the temperature of liquid nitrogen, freeze-dried, and treated with formaldehyde gas for the histochemical demonstration of adrenergic nerves (Falck, 1962; Falck, Hillarp, Thieme \& Torp, 1962; Corrodi \& Jonsson, 1967; for further methodological details, see Falck \& Owman, 1965). Serial transverse sections of $6 \mu \mathrm{m}$ thickness were prepared for fluorescence microscopy. Under 
the optical conditions used, the noradrenaline fluorophore exhibits an intense green fluorescence.

(2) Hypogastric denervation was performed as above in fourteen male rats, and fourteen intact males served as controls. After decapitation, the region from the bladder and adjoining urethra described above was dissected out and homogenized in $0.4 \mathrm{~N}$-perchloric acid for the spectrofluorometric determination of noradrenaline according to the method of Bertler, Carlsson, Rosengren \& Waldeck (1958), as modified by Häggendal (1963). Tissues from one animal were used for each determination.

(3) Fifteen male rats were subjected to hypogastric denervation as above. One week later, ten of these males were placed overnight in separate cages together with three females for mating. The females were all in the prooestrus stage, as checked microscopically on vaginal smears stained with haematoxylin-eosin. On the following day, new vaginal smears were taken and analysed microscopically for the presence of spermatozoa. The males were killed and the contents of the urinary bladder were spread on a microscope slide, which was dried in air and stained in haematoxylin-eosin for the detection of any spermatozoa. In order to exclude any influence of the operative trauma on the sexual activity, ten males were subjected to sham-operation followed by the same mating procedure. The remaining five males with hypogastric denervation were not given access to females; their bladder contents were similarly examined for the presence of any spermatozoa 1 week after operation.

\section{Guinea-pig experiments}

A total of twenty-two male guinea-pigs weighing approximately $400 \mathrm{~g}$ were used. The animals were allowed unrestricted access to standard pellets (SANbolagen, Sweden), turnips and tap water.

(1) In eight animals, about $35 \mathrm{~mm}$ of both hypogastric nerves were resected according to the operation procedure described for the rats. Eight intact animals served as controls. The lower part of the urinary bladder including the proximal portion of the urethra, i.e. the same region as described for the rat, was dissected out for chemical determination of noradrenaline (see above). One piece of tissue was used for each determination.

(2) Three animals were anaesthetized with nembutal $(30 \mathrm{mg} / \mathrm{kg}$ intraperitoneally) supplemented with ether when necessary. The abdomen was opened by a midline incision and the hypogastric nerves were carefully freed. The nerves were hooked on a bipolar, insulated platinum electrode and covered with the abdominal viscera to prevent drying. Each vas deferens was exposed, and $0.02 \mathrm{ml}$ India ink was deposited through a fine cannula in the lumen on each side of its most distal part. During stimulation of the nerves $(50 \mathrm{~V}$, duration $2 \mathrm{msec}$, frequency $15 / \mathrm{sec}$ ), the transport of the India ink in a proximal direction was noted through the translucent wall of the vas deferens.

(3) Another three animals were subjected to laparotomy under ether anaesthesia, and $0.02 \mathrm{ml}$ India ink was deposited in the most distal part of each vas deferens as above. The abdominal incision was closed and the animal allowed to recover. Three days later, the vasa deferentia were again exposed for 
inspection of the position of the India ink deposit through their translucent wall.

\section{Studies on human tissue}

Pieces of presacral connective tissue were obtained from four male patients aged 56 to 64 years. The tissues were frozen to the temperature of liquid nitrogen and further processed for demonstration by the formaldehyde fluorescence technique of any adrenergic structures present (for method, see above).

\section{RESULTS}

\section{Rat experiments}

(1) Fluorescence microscopy of the preparations from the urinary bladder revealed the presence of characteristically beaded adrenergic nerve terminals, both in the smooth musculature and around blood vessels. The entrance of the ureters was easily recognized when they penetrated obliquely through the bladder wall. The distal portion of the ureteric wall contained a fairly small but substantial number of fluorescent nerve terminals running in the muscular layer, mainly in a circular manner. In its most cranial part, the trigonum of the bladder contained only very few adrenergic nerves in the musculature (Pl. 1, Fig. 1) and they were strictly separated from the ureteric nerves. Most of the nerves in this region of the trigonum were vascular. More caudally, the number of muscular nerves in the trigonum area increased (Pl. 1, Fig. 2) and they then became mixed with the nerves supplying the ureters. Further below the ureteric ostia, i.e. in the internal sphincter of the bladder, the adrenergic innervation in the well developed smooth muscle layers was very dense (Pl. 1, Fig. 3). The nerves ran in intimate relation to the muscle fibres, preferably parallel to the underlying mucosa. The density of adrenergic muscular innervation was also high more caudally, i.e. in the most proximal portion of the urethra, where the nerve terminals had the same arrangement as in the adjacent trigonum area. More distally in the urethra, at the level of the colliculus seminalis, the number of green-fluorescent nerves in the smooth musculature remained essentially unchanged (Pl. 2, Fig. 4). A somewhat smaller amount of adrenergic nerve terminals occurred in the smooth muscle wall of the urethra distal to the colliculus seminalis (Pl. 2, Fig. 5).

Intramural ganglia were not recognized in any part of the bladder preparations taken for fluorescence microscopic analysis.

\section{EXPLANATION OF PLATE 1}

Fluorescence photomicrographs showing transverse sections of the rat urinary bladderurethra. The mucosa is in the upper part of the pictures. $\times 100$.

FIG. 1. Bladder trigonum at the level of the entrance of the ureters. Only few greenfluorescent adrenergic nerve terminals are seen in the smooth musculature. Small vessels with adrenergic innervation in the upper part of the picture.

FIG. 2. An increased number of nerve terminals occur in the trigonum somewhat caudally to the ureteric entrances.

FIG. 3. Well below the ureteric ostia, i.e. in the internal sphincter of the bladder, the smooth musculature receives a very dense adrenergic nerve supply. 

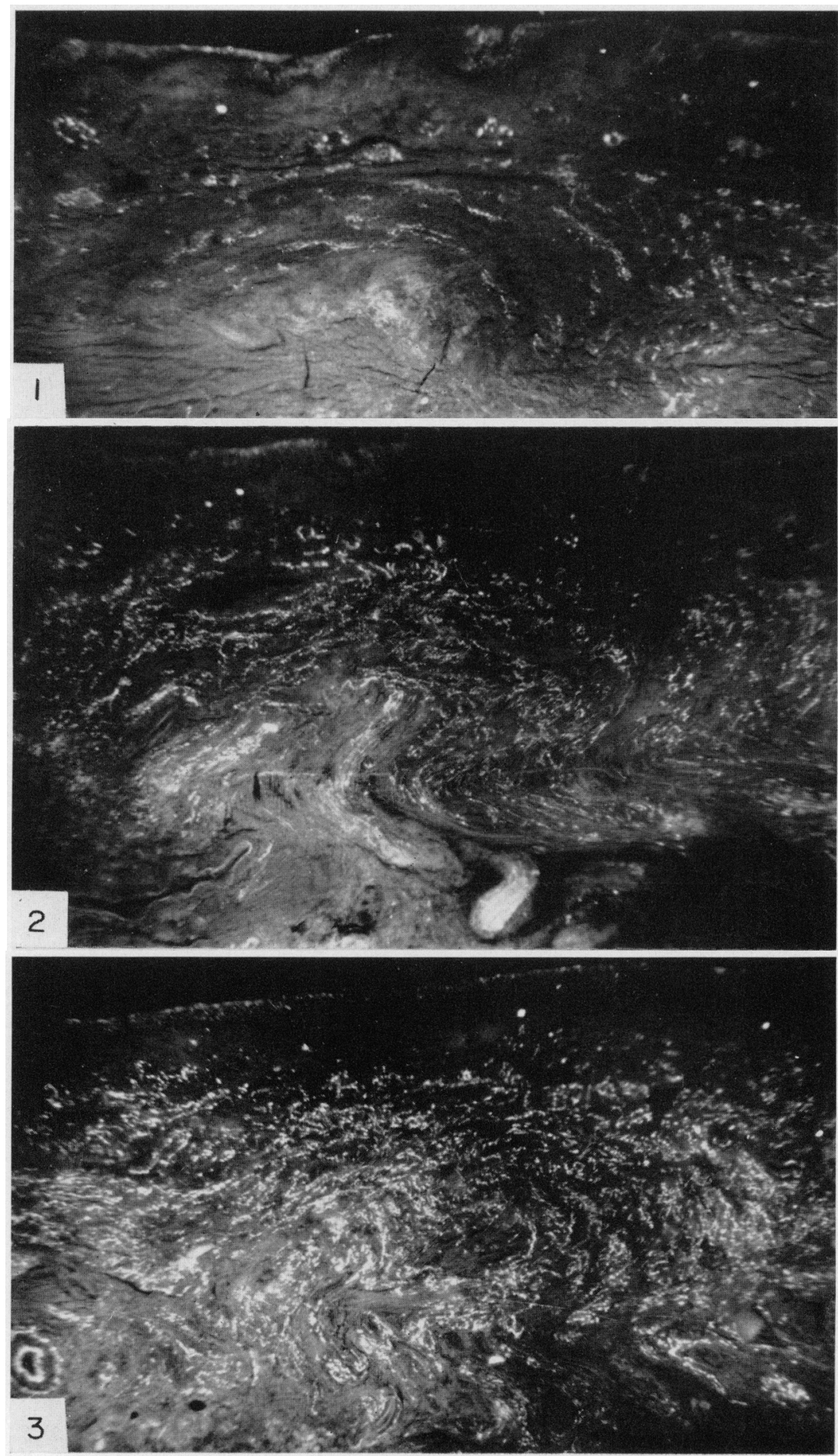

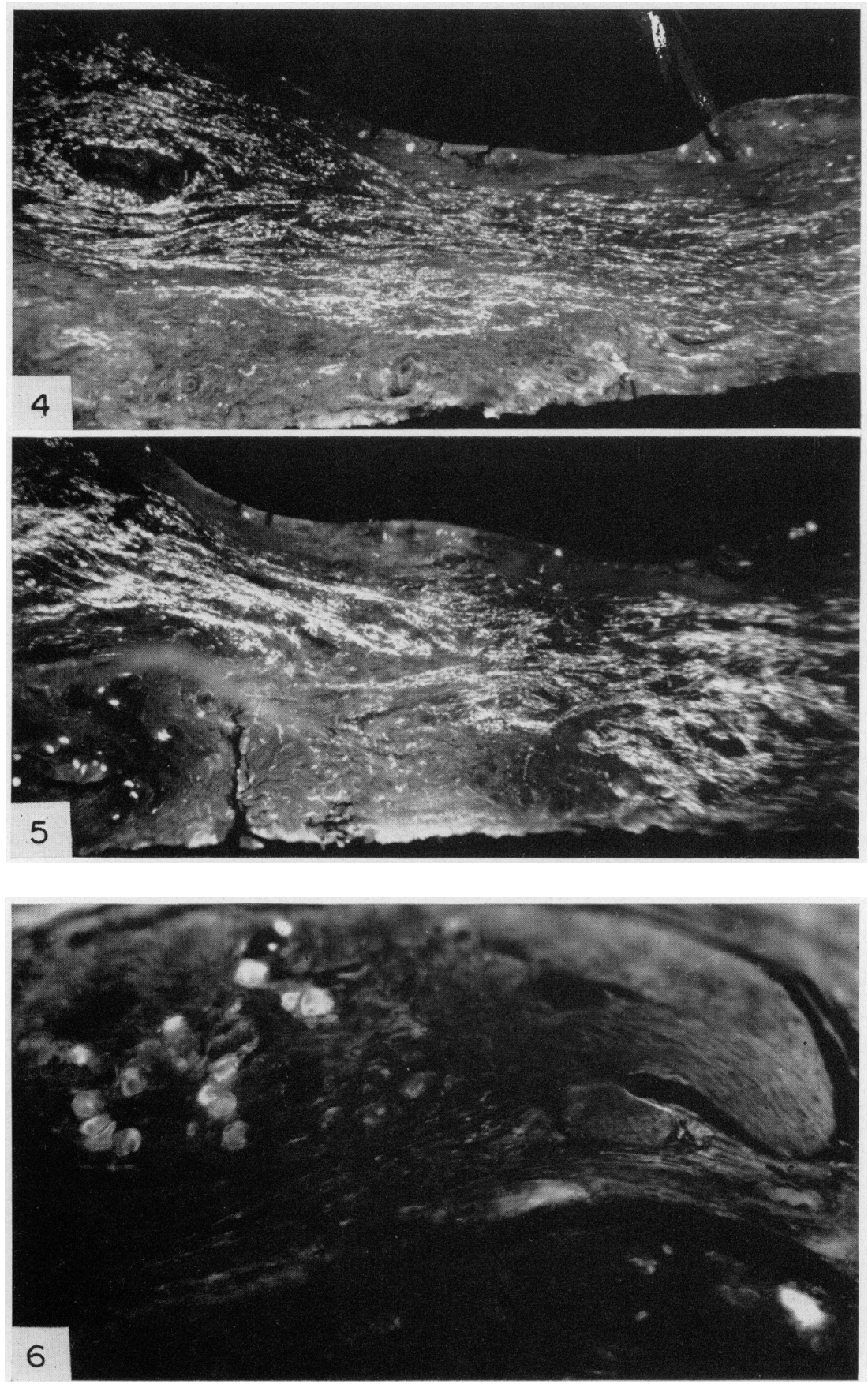
Hypogastric denervation did not overtly affect the number of fluorescent nerve terminals in any region of the bladder trigonum or the urethra.

(2) The concentration of noradrenaline in the lower part of the bladder including its neck and the proximal portion of the urethra was $7.82 \pm 0.54$ $\mu \mathrm{g} / \mathrm{g}$ wet weight (mean \pm S.E.M.) in the controls. One week after hypogastric denervation, the concentration was $6 \cdot 76 \pm 0 \cdot 64 \mu \mathrm{g} / \mathrm{g}$, which was not statistically different from that of the controls (Student's $t$ test: $P>0 \cdot 05$ ).

(3) The results of the mating experiments were very consistent. Thus, in the series with males which had been subjected to sham operation, spermatozoa were always detected in the vaginal smear from at least one of the three females kept in the same cage. Spermatozoa were never detected in the bladder content of these males. By contrast, spermatozoa were always present-sometimes in large numbers-in the urinary bladder of males mating after hypogastric denervation. In these series, spermatozoa were consistently absent from the vaginal smears of the corresponding female animals. In the males subjected to hypogastric denervation but maintained entirely separate from females, spermatozoa were regularly found in the contents of the urinary bladder.

\section{Guinea-pig experiments}

(1) As in the rats, hypogastric denervation did not affect the level of noradrenaline in the lower part of the bladder, including the proximal urethra $(P>0 \cdot 05)$. The concentration was $3 \cdot 30 \pm 0.44 \mu \mathrm{g} / \mathrm{g}$ compared with that in the intact controls which was $2.95 \pm 0.38 \mu \mathrm{g} / \mathrm{g}$.

(2) Stimulation of the hypogastric nerves resulted in an immediate and vigorous contraction in the vasa deferentia and the seminal vesicles. Within seconds, the India ink deposit was transported to the most proximal portion of the vasa deferentia and, concomitantly, opalescent fluid was seen to extrude from the external urethral meatus. At autopsy, seminal fluid contaminated with India ink was present in the entire length of the urethra, whereas India ink was absent from the contents of the bladder.

(3) The spontaneous proximal transport of India ink was studied 3 days after its deposition in the most distal part of the vasa deferentia. A greyish luminal content, obviously reflecting diluted India ink, was visible through the translucent wall from the point of injection and extended about $40 \mathrm{~mm}$ in a proximal direction.

\section{EXPLANATION OF PLATE 2}

Fluorescence photomicrographs (Figs. 4 and 5) of rat urinary bladder-urethra as in Plate 1.

FIG. 4. The adrenergic innervation is high also in the urethra at the level of the seminal collicle. $\times 100$.

FIG. 5. More distally in the urethra, the number of nerve terminals in the musculature is somewhat decreased. $\times 100$.

Fic. 6. Tissue removed at operation in the presacral region of a male patient aged 58 years. The preparation contains a ganglion formation (left half of picture) consisting of both green-fluorescent adrenergic and non-fluorescent (probably cholinergic) ganglia. A thick bundle of smooth, non-fluorescent nerve fibres is seen to leave the ganglion (upper right half of the picture). A couple of small, intensely green-fluorescent cells are shown in the right lower corner. $\times 200$. 
Observations on human tissues

All tissue obtained from the presacral region contained thick bundles of nerve fibres visible through their faint, unspecific autofluorescence ( $\mathrm{Pl}$. 2, Fig. 6). In addition, some of the preparations contained groups of characteristic ganglion cells exhibiting a green formaldehyde-induced fluorescence of moderate intensity in their cytoplasm (Pl. 2, Fig. 6). The fluorescence could sometimes be seen to extend for a short distance into the most proximal part of the axon. Small, intensely green-fluorescent cells with one or more short processes were usually observed in small clusters adjacent to the adrenergic ganglion cells.

\section{DISCUSSION}

Langley \& Anderson (1895a) demonstrated that the motor response of the vas deferens and the seminal vesicles in cats and rabbits is transmitted along a pathway running from the lumbar sympathetic outflow, through the inferior mesenteric ganglion and hypogastric nerves. In conformity with these findings, Semans \& Langworthy (1938) showed that the emission of seminal fluid is controlled by the hypogastric nerves. It has long been known (Remy, 1886) that transection of the hypogastric nerves results in sterility in the male guineapig. These findings were confirmed by Simeone (1933). A similar effect was obtained in rabbits after bilateral lumbar sympathectomy (Bacq, 1931). In humans, Learmonth (1931) systematically stimulated the individual roots of the presacral (hypogastric) nerve, and confirmed the sympathetic origin of the innervation to the male accessory genital apparatus, including the posterior urethra and the bladder neck. From denervation studies on rabbits, Hodson (1964, 1965) suggested that the sterility produced by interference with the sympathetic innervation to the pelvic organs is largely due to reflux of the semen into the bladder as a result of a permanent paralysis of the bladder neck, whereas the contractile function of the smooth musculature concerned with seminal emission was claimed to recover progressively after denervation.

The present study revealed a considerable amount of noradrenaline in the region of the bladder neck (including the trigonum and the posterior urethra) of male guinea-pigs and rats. This agreed with an ample adrenergic innervation of the smooth musculature, shown histochemically in the rat. Removal of the hypogastric nerves did not significantly affect the level of noradrenaline or the number of adrenergic nerves in these tissues, indicating the presence of a peripheral synapse. This agrees with earlier findings of adrenergic ganglion cells in the terminal part of the hypogastric nerve where it joins the vessels to the accessory genital organs (Falck, Owman \& Sjöstrand, 1965; Owman \& Sjöstrand, 1965) and close to the proximal part of the urethra (Owman, Owman \& Sjöberg, 1971). The findings indicate that the region of the bladder neck in guinea-pigs and rats receives its postganglionic adrenergic innervation mainly, if not entirely, from short adrenergic neurons rather than from the hypogastric nerves. The organization appears to be somewhat different in the cat (Hamberger \& Norberg, 1965a, b), in which the hypogastric nerves carry a significant portion of postganglionic nerves to the bladder trigonum which, in addition, contains intramural adrenergic ganglion cells. 
It was confirmed that electrical stimulation of the hypogastric nerves produces a vigorous contraction of the vasa deferentia and the seminal vesicles, resulting in proximal transport of the contents of the lumen of the vas into the proximal urethra. At the same time, nothing entered the bladder. This emission response also resulted in the extrusion of fluid through the external urethral meatus, though not in the form of the jets which characterize ejaculation. Reflux of semen into the urinary bladder after hypogastric denervation with consequent absence of spermatozoa from the vagina upon mating was also confirmed. After hypogastric denervation, however, spermatozoa still entered the bladder even though the male was not allowed to copulate. This was in agreement with the direct finding that a proximal, though slow, transport of the luminal contents in the vas deferens persisted after hypogastric denervation. In 1933, Simeone had observed that a powerful segmental contraction occurred in the ductus epididymidis after hypogastric denervation. Such a reduced flow of the luminal contents conceivably accounts for the pronounced enlargement of the vasa deferentia and the seminal vesicles which has been frequently observed after sympathetic denervation (Remy, 1886; Bacq, 1931; Simeone, 1933; Hodson, 1964, 1965).

May, DeWeese \& Rob (1969) and Hallböök \& Holmquist (1970) have shown that extensive operative dissection in the presacral region of male patients in connection with reconstruction of the distal aorta and the common iliac arteries results in a reflux of seminal fluid into the bladder. Preparations obtained from such operations revealed the presence of adrenergic ganglion formations which may be identical with those supplying the pelvic organs, including the bladder neck, with short adrenergic neurons.

From the present results, it appears that the short adrenergic neurons constitute the final link in the sympathetic innervation of the smooth musculature in structures engaged in the emission mechanism, including the vasa deferentia, seminal vesicles, prostate and the bladder neck region. The ganglia are supplied by the hypogastric nerves. Removal of these ganglia, the hypogastric nerves, or more proximal sympathetic pathways results in a permanent motor deficiency of the above-mentioned structures. The seminal fluid is continuously transported through the vasa deferentia, although at a very slow rate, due to a segmental contraction and a permanent secretion from the accessory genital glands. Postoperative recovery of the motor function in the vasa deferentia and the seminal vesicles is not, therefore, a prerequisite for a seminal reflux into the bladder. This reflux is continuous and occurs independently of copulation. It would seem contradictory that patients having a seminal fluid reflux into the bladder usually observe seminal contamination of the urine only after coitus. However, it is possible that the persistent post-denervation reflux is small and therefore escapes detection whereas contraction of surrounding pelvic musculature during coitus promotes the passive transport in the internal genital organs, followed by a more voluminous reflux.

\section{ACKNOWLEDGMENT}

Supported by a grant from the Ford Foundation (No. 68-383), New York. 


\section{REFERENCES}

BACQ, Z. M. (1931) Impotence of the male rodent after sympathetic denervation of the genital organs. Am. J. Physiol. 96, 321.

Baumgarten, H. G., Falck, B., Holstein, A.-F., Owman, Ch. \& Owman, T. (1968) Adrenergic innervation of the human testis, epididymis ductus deferens and prostate: a fluorescence microscopic and fluorimetric study. Z. Zellforsch. mikrosk. Anat. 90, 81.

Bertuer, Å., Garlsson, A., Rosengren, E. \& Waldeck, B. (1958) A method for the fluorimetric determination of adrenaline, noradrenaline and dopamine in tissues. $K$. fysiogr. Sällsk. Lund Förh. 28, 121.

Corrodi, H. \& Jonsson, G. (1967) The formaldehyde fluorescence method for the histochemical demonstration of biogenic monoamines. F. Histochem. Cytochem. 15, 65.

FALck, B. (1962) Observations on the possibilities of the cellular localization of monoamines by a fluorescence method. Acta physiol. scand. 56, Suppl. 197, 1.

Falck, B., Hillarp, N.-A., Thieme, G. \& Torp, A. (1962) Fluorescence of catechol amines and related compounds condensed with formaldehyde. 7. Histochem. Cytochem. 10, 348.

Falck, B. \& Owman, Ch. (1965) A detailed methodological description of the fluorescence method for the cellular demonstration of biogenic monoamines. Acta Univ. lund. II. 7, 1.

Falck, B., Owman, Ch. \& Sjöstrand, N. O. (1965) Peripherally located adrenergic neurons innervating the vas deferens and the seminal vesicle of the guinea-pig. Experientia, 21, 98.

Gennser, G., Owman, Gh., Owman, T. \& Wehlin, L. (1969) Significance of adrenergic innervation of the bladder outlet during ejaculation. Lancet, i, 154.

Goligher, J. G. (1951) Sexual function after excision of the rectum. Proc. R. Soc. Med. 44, 824.

HÄGGENDAL, J. (1963) An improved method for fluorimetric determination of small amounts of adrenaline and noradrenaline in plasma and tissues. Acta physiol. scand. 59, 242.

Hallböök, T. \& HolmQuist, B. (1970) Sexual disturbances following dissection of the aorta and the common iliac arteries. F.cardiovasc. Surg. 11, 255.

HAMBERger, B. \& NorberG, K.-A. (1965a) Adrenergic synaptic terminals and nerve cells in bladder ganglia of the cat. Int. F. Neuropharmacol. 4, 41.

HAMBERGER, B. \& NORBERG, K.-A. (1965b) Studies on some systems of adrenergic synaptic terminals in the abdominal ganglia of the cat. Acta physiol. scand. 65, 235.

Hodson, N. (1964) Role of the hypogastric nerves in seminal emission in the rabbit. F. Reprod. Fert. 7, 113.

Hodson, N. (1965) Sympathetic nerves and reproductive organs in the male rabbit. F. Reprod. Fert. 10, 209.

Hotchiss, R. S., Pinto, A. B. \& Kleggman, S. (1955) Artificial insemination with semen recovered from the bladder. Fert. Steril. 6, 37.

LANGley, J. N. \& Anderson, H. K. (1895a) The innervation of the pelvic and adjoining viscera. Part 4. The internal generative organs. F. Physiol., Lond. 19, 122.

Langley, J. N. \& Anderson, H. K. (1895b) The innervation of the pelvic and adjoining viscera. Part 5. Position of the nerve cells on the course of the efferent nerve fibres. F. Physiol., Lond. 19, $13 \mathrm{I}$.

Langley, J. N. \& Anderson, H. K. (1896) The innervation of the pelvic and adjoining viscera. Part 7. Anatomical observations. F. Physiol., Lond. 20, 372.

LEARmonth, J. R. (1931) A contribution to the neurophysiology of the urinary bladder in man. Brain, 54, 147.

MAY, A. G., DeWeese, J. A. \& RoB, G. G. (1969) Changes in sexual function following operation on the abdominal aorta. Surgery, St. Louis, 65, 41.

Owman, Сh., Owman, T. \& SJöberg, N.-O. (1971) Short adrenergic neurons innervating the female urethra of the cat. Experientia, 27, 313.

Owman, Сн. \& SJöstrand, N. O. (1965) Short adrenergic neurons and catecholamine-containing cells in vas deferens and accessory male genital glands of different mammals. Z. Zellforsch. mikrosk. Anat. 66, 300.

Remy, C. (1886) Nerfs éjaculateurs. J. Anat. Physiol., Paris, 22, 205.

RIESER, C. (1961) The etiology of retrograde ejaculation and a method for insemination. Fert. Steril. 12, 488.

Schellen, T. M. C. M. (1960) A case of retrograde ejaculation caused by a colon operation. Fert. Steril. $11,187$.

Semans, J. H. \& LANGworthy, O. R. (1938) Observations on the neurophysiology of sexual function in the male cat. F. Urol. 40, 836.

Simeone, F. A. (1933) A neuromuscular mechanism in the ductus epididymidis and its impairment by sympathetic denervation. Am. F. Physiol. 103, 582. 
SJöstrand, N. O. (1962a) Inhibition by ganglionic blocking agents of the motor response of the isolated guinea-pig vas deferens to hypogastric nerve stimulation. Acta physiol. scand. 54, 306.

SJöSTRAND, N. O. (1962b) Effect of reserpine and hypogastric denervation on the noradrenaline content of the vas deferens and seminal vesicle of the guinea-pig. Acta physiol. scand. 56, 376.

SJöstrand, N. O. (1965) The adrenergic innervation of the vas deferens and the accessory male genital glands. Acta physiol. scand. 65, Suppl. 257, 1.

Walters, D. \& Kaurman, M. S. (1959) Sterility due to retrograde ejaculation of semen. Am. 7 . Obsiet. Gynec. 78, 274. 